\title{
GERINNUNGSSPRECHSTUNDE
}

\author{
Ihr Patient braucht Antithrombotika
}

\section{Beinvenenthrombose: wie und wie lange antikoagulieren?}

\author{
Immer mehr Patienten erhalten eine antithrombotische Therapie - sei \\ es wegen Venenthrombose, Herzklappenersatz oder Vorhofflimmern. In \\ dieser "Gerinnnungssprechstunde" erhalten Sie Antworten auf Fragen, \\ die sich dabei im hausärztlichen Alltag ergeben.
}

\section{? Nachdem alle vier neuen oralen Antikoagulanzien (NOAKs) auch für die Therapie der tiefen Beinvenen- thrombose (TVT) bzw. Lungenembolie (LE) zugelassen sind, fragt sich, ob in solchen Situationen überhaupt noch eine initiale Injektionstherapie mit einem Heparin nötig ist. Kann man nicht direkt mit einem NOAK starten?}

Diese Frage muss man differenziert bzw. präparatespezifisch beantworten. Das Design der Zulassungsstudien war mit den vier Substanzen unterschiedlich. Deshalb sollte man so vorgehen, wie dies in den Studien festgelegt wurde.

Entscheiden Sie sich für Rivaroxaban, können Sie direkt mit dieser Substanz nach Diagnosesicherung starten, wobei in den ersten drei Wochen $2 \times 15 \mathrm{mg}$ täglich gegeben werden, danach nur noch $1 \times 20 \mathrm{mg}$ täglich.

Auch bei Apixaban können Sie sofort mit der oralen Therapie beginnen. Die ersten sieben Tage werden $2 \times 10 \mathrm{mg}$ täglich gegeben, ab dem 8 . Tag $2 \times 5 \mathrm{mg}$.

Entsprechend dem Design der jeweiligen Zulassungsstudie müssen Sie bei Edoxaban und Dabigatran die Therapie zunächst mit einem Heparin-Präparat, i. d. R. einem niedermolekularen Heparin (NMH) in voller therapeutischer Dosierung beginnen und nach fünf Tagen auf das NOAK, nämlich 1 x $60 \mathrm{mg}$ Edoxaban oder 2 x $150 \mathrm{mg}$ Dabigatran switchen. Für alle NOAKs gilt, dass die Dosierung bei einer stärkeren Einschränkung der Nierenfunktion oder einem hohem Blutungsrisiko angepasst werden muss.
Egal, für welche Substanz Sie sich entscheiden, es gibt immer eine Änderung in der Medikation, entweder von einem NMH auf Edoxaban oder Dabigatran oder in der Dosierung des NOAK bei Rivaroxaban oder Apixaban. Diese Schnittstellen muss man sorgfältig im Auge behalten.

$?$ Wie muss man NMH bei Patienten mit einer chronischen Niereninsuffizienz dosieren?

Alle NMH werden überwiegend renal eliminiert und können bei chronischer Niereninsuffizienz kumulieren. Bis zu einer GFR von $30 \mathrm{ml} / \mathrm{min}$ können alle $\mathrm{NMH}$ in voller Dosierung gegeben werden. Eine Dosisanpassung ist nicht nötig. Bei einer GFR $<30 \mathrm{ml} / \mathrm{min}$ muss die Dosis von Enoxaparin halbiert werden. Für Tinzaparin konnte in einer Studie gezeigt werden, dass bei einer GFR von 20-29 $\mathrm{ml} / \mathrm{min}$ über zehn Tage keine Akkumulation auftritt. Und bei Certoparin sollte die Dosierung bei einer GFR $<30$ $\mathrm{ml} / \mathrm{min}$ auf $3.000 \mathrm{IE} /$ Tag reduziert werden. Für Dalteparin wird auch bei einer GFR $<30 \mathrm{ml} / \mathrm{min}$ keine Dosisanpassung empfohlen, aber eine erhöhte Vorsicht. $\mathrm{Ab}$ einer GFR von $30 \mathrm{ml} / \mathrm{min}$ sind Reviparin und Nadroparin kontraindiziert.

Die Empfehlungen der Kliniken bzgl. der Dauer einer oralen Antikoagulation bei Patienten mit einer TVT bzw. LE sind sehr unterschiedlich, um nicht zu sagen verwirrend: einmal drei Monate, dann sechs Monate, bei

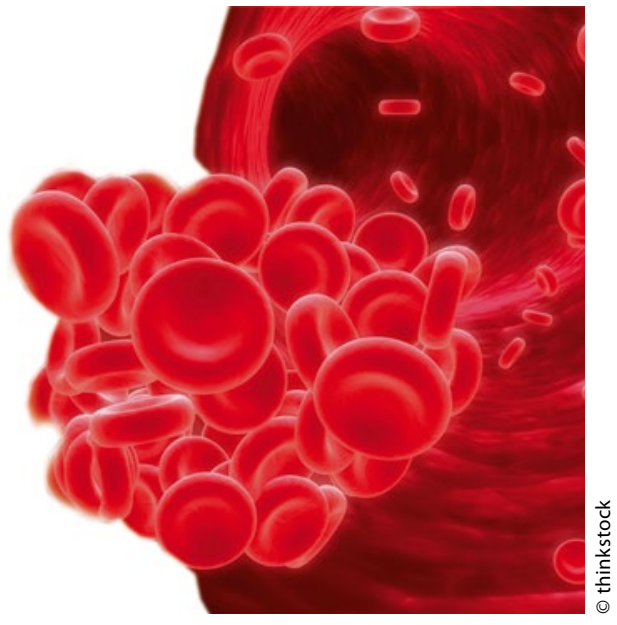

anderen Patienten wiederum dauerhaft. Wie soll ich mich verhalten?

Sie haben Recht. Die Empfehlungen bzgl. Dauer einer oralen Antikoagulation (OAK) nach einer TVT oder LE sind heute viel individueller als früher. Grundsätzlich sollten Patienten mit einer provozierten Thrombose mit oder ohne LE, z.B. nach einem operativen Eingriff oder bei einer Immobilisation der unteren Extremitäten, mindestens drei Monate antikoaguliert werden. Auch bei einer spontanen Thrombose mit oder ohne LE wird eine Antikoagulation von drei Monaten empfohlen. Bei Patienten mit einem zweiten Ereignis sollte jedoch immer eine dauerhafte OAK erfolgen. Dies gilt auch für Patienten mit einem erhöhten Rezidivrisiko. Dazu gehören insbesondere solche Patienten, bei denen einige Wochen nach Absetzen der OAK erhöhte DDimere nachgewiesen werden. Gleiches gilt auch für Tumorpatienten, die allerdings initial nach dem ersten Ereignis immer über mindestens sechs Monate mit einem NMH behandelt werden sollten.

Eine über drei Monate hinausgehende Antikoagulation bei einem Erstereignis ist jedoch nur dann zu vertreten, wenn kein erhöhtes Blutungsrisiko vorliegt und auch während der ersten drei Monate keine Komplikation aufgetreten ist. Entscheidet man sich für eine verlängerte Antikoagulation, so sollte in regelmäßigen Abständen das individuelle Nutzen-Risiko-Verhältnis immer wieder neu ermittelt werden.

Dr. Peter Stiefelhagen 